\title{
Coordination and Collaboration
}

National Cancer Institute

\section{Source}

National Cancer Institute. Coordination and Collaboration. NCI Thesaurus. Code C18724.

Support research for the discovery, development, and evaluation of strategies for preventing or minimizing the negative physical, psychological, cognitive, and social consequences of HIV, including stigmatization of persons with or at risk for HIV infection. Support research strategies for promoting effective health care utilization among persons with HIV infection. 